\title{
Theory and application of the equivalent transformation relationship between plane wave in one-dimensional medium and cylindrical wave in polar geometry
}

\author{
Y. Wang*, Z.L. Yang**, J.W. Zhang*** \\ *College of Aerospace and Civil Engineering, Harbin Engineering University, Harbin, China, \\ E-mail: wangyao-1234567@hotmail.com \\ **College of Aerospace and Civil Engineering, Harbin Engineering University, Harbin, China, \\ E-mail: yangzailin00@163.com \\ ***College of Aerospace and Civil Engineering, Harbin Engineering University, Harbin, China, \\ E-mail: zhang_1.2005@163.com
}

cross $^{\text {ref }}$ http://dx.doi.org/10.5755/j01.mech.21.3.8961

\section{Introduction}

The cylindrical wave is a common type of wave motion in homogeneous medium [1]. In general, the cylindrical wave is caused by the linear source, such as a cylindrical hole subjected to a time-varying uniform internal pressure. The group velocity of elastic wave is constant in the homogeneous medium, so that the wave travels the same distance in the equal time internal. Then, the plane travelling wave's amplitude is constant in the homogeneous medium, but the cylindrical wave's amplitude reduces with the travelling distance.

The situation is usually different in the inhomogeneous medium. The functionally graded material is a classical inhomogeneous medium. In the process of material production, the ratios of the components are controlled to be distributed continuously. Thus, the macroscopic material properties are graded. Therefore, the amplitude and group velocity of the travelling wave may both vary in the process of travelling.

Because of the material's natural inhomogeneity, the old concepts, theories, algorithms and experimental measures which are introduced and developed for homogeneous materials to meet great difficulty. A lot of them are no longer suitable for inhomogeneous media, so that more works are needed for the research method of the inhomogeneous medium. The behaviour of the inhomogeneous medium is a very hot topic. In order to investigate the behaviours of the inhomogeneous medium with gradient material properties, a lot of exploration and innovation have already been done. For example, Kim and Paulino [2] proposed the graded finite elements for continuously nonhomogeneous isotropic and orthotropic materials within the framework of a generalized isoparametric formulation. Such elements possess a spatially-varying material property field, e.g. Young's modulus $E$ and Poisson's ratio $v$ for isotropic materials; and principal Young's modulus $E_{11}$, $E_{22}$, in-plane shear modulus $G_{12}$, and Poisson's ratio $v_{12}$ for orthotropic materials.

The wave propagation in inhomogeneous media is a classical topic in theories of acoustic, electromagnetic, elastic and seismic waves. Many naturally-occurring soils, such as flocculated clays, varved silts or sands, are typically deposited via sedimentation over long periods. The effects of deposition, overburden, and desiccation can cause soil media to exhibit both anisotropic and inhomogeneous deformability [3]. The wave motion problem in the inhomogeneous medium is normally solved by using numerical techniques, such as reverberation matrix method, finite difference method and finite element method. Pao and others [4] presented the reverberation matrix method for wave propagation in a multi-layered liquid. Virieux [5, 6] presented a finite-difference method for modelling $\mathrm{SH}$ and P-SV wave propagation in heterogeneous media by using velocity and stress in a discrete grid. The finite difference formulation derived by strong form of wave equation with coefficient variations is efficient and accurate for the wave motion simulation of inhomogeneous media with gradient material properties. However, the finite element method is more widely used, and easier to implement on computer for engineering than the finite difference method. Oyekoya and others [7] developed the Mindlin-type element and Reissner-type element for the modelling of FGC plate subjected to buckling and free vibration. Yang and Wang $[8,9]$ proposed the dynamic inhomogeneous finite element method for the dynamic response analysis of the inhomogeneous media. The material properties in dynamic inhomogeneous finite element are graded, so that the error of discretization and the scale of calculation can be reduced greatly. In addition, the wave propagation in the inhomogeneous infinite domain should be simulated after dealing with the exterior problem. The techniques can be categorized into three types: boundary dampers, both plane and cylindrical (also called non-reflecting boundary conditions); linking to exterior solutions, both series and boundary integral (also called Dirichlet to Neumann mapping); infinite elements. The most widely used method is boundary damper, such as the perfectly matched layer (PML), or 'sponge layer'. The idea of PML is that the outgoing wave is not absorbed on a boundary, but in a domain which extends beyond the boundary. In this domain the wave is absorbed or damped in such a way that it does net return into the computational domain [10]. Kucukcoban and Kallivokas [11] discussed a mixed displacement-stress formulation for forward elastic wave simulations in PML truncated heterogeneous media.

There is great difficulty to obtain analytic solution for wave motion problem in the inhomogeneous medium with the material property field. The worldwide scholars did a lot of analytical and numerical works, but only a few 
Typical examples of material property fields in published papers

\begin{tabular}{|c|c|}
\hline Types of inhomogeneity & Author \\
\hline$G=G_{0} e^{c z}$ & Wilson [12] \\
\hline$G(z)=G_{0}+\left(G_{\infty}-G_{0}\right)\left(1-e^{\alpha z}\right), \quad 0<G_{0} \leq G_{\infty}$ & Vrettos $[13,14]$ \\
\hline$G(z)=G_{0}+\left(G_{\infty}-G_{0}\right)\left(1-e^{-\alpha z}\right)$ and $G(z)=G_{0}(1+b z)^{1 / 3}$ & Vrettos [15] \\
\hline$G(z)=G_{0}+\left(G_{\infty}-G_{0}\right)\left(1-e^{\alpha z}\right)$ and $G(z)=G_{0}(1+m z)$ & Leung et al. [16] \\
\hline$G=G_{2}+G_{1} z$ & Stoneley [17], Newlands [18] \\
\hline$G=G_{0}(1+0.5 \delta z), G=G_{0} \sqrt{1+\delta z}$ & Vardoulakis [19] \\
\hline$v_{p}(z)=v_{p 0}\left(1+\frac{2 \bar{\alpha}}{v_{p 0}} Z\right)^{-1 / 2}, v_{s}(z)=v_{s 0}\left(1+\frac{2 \bar{\beta}}{v_{s 0}} Z\right)^{-1 / 2}$ & Ben-Menahem [20] \\
\hline$\mu=\mu^{\prime}\left(1+\frac{z-h}{\lambda}\right)^{2}$ & Hudson [21] \\
\hline$G / G_{0}=\left(z / z_{0}\right)^{a}, \quad \rho / \rho_{0}=\left(z / z_{0}\right)^{a-2}$ & Hook [22] \\
\hline $\begin{array}{cl}\mu=\mu_{0}(1+z / \lambda)^{2}, & \rho=\rho_{0}(1+z / \lambda)^{2} \\
\mu=\mu_{0} e^{\alpha z}, & \rho=\rho_{0} e^{\beta z}\end{array}$ & Deresiewicz [23] \\
\hline$G(z)=G_{0}(1+b z)^{2}$ & Gazetas [24] \\
\hline$\mu=\mu_{0}(r / a)^{\lambda}, \quad \rho=\rho_{0}(r / a)^{\lambda-2}$ & Watanabe [25] \\
\hline$k(z)=k_{0}(1+A Z)^{1 / 2}$ & Manolis and Bagtzoglou [26] \\
\hline$\mu=\mu_{0}(1+k y / h)^{p}, \quad \rho=\rho_{0}(1+k y / h)^{p-2}$ & Watanabe and Payton [27] \\
\hline$\rho / \rho_{0}=\mu / \mu_{0}=A \cosh ^{2}(\alpha+\beta z)$ & Pekeris [28] \\
\hline$A(z)=\alpha_{0}+\beta_{0} z+\sum_{j=1}^{M} \alpha_{j} e^{-\gamma_{j} z}$ & Shaw and Makris [29] \\
\hline$G=G_{0}(1+b z)^{2 n}, \quad \rho=\rho_{0}(1+\alpha z)^{m}$ & Dey et al. [30] \\
\hline
\end{tabular}

forms of material property distribution have been investigated and solved. Some typical examples are shown in Table 1.

There are some interesting techniques and applications in the behaviour researches of the inhomogeneous medium, such as the homogenization theory (Boutin and Auriault [31], Chen and Fish [32], Nicolas [33], Fish and others [34]) and wave cloak (Pendry et al. [35], Zhou et al. [26], McManus et al. [37]). Homogenization aims at deriving a homogenized description (governing partial differential equations and constitutive law) for the medium, based on the assumption that a statistically homogeneous medium represented by a "representative volume element" (RVE), or a material with periodic structure represented by a "repeated unit cell" (RUC), can be defined. Mathematical homogenization provides a rigorous definition of the homogenization process and the homogenized equations. It consists of setting the problem as a sequence of equations describing the heterogeneous material [33]. Using the freedom of design that metamaterials provide, wave fields can be redirected at will and propose a design strategy. Metamaterials which owe their properties to subwavelength details of structure rather than to their chemical composition, can be impossible to find in nature [35]. We can design their properties based on our purposes. These two techniques mentioned above show that there are some equivalent transformation relations between the microstructure and properties of material, which are of great significance and value in the theoretical study and engineering applications.

The present paper investigates the equivalent transformation relations between the plane wave in 1D medium and the cylindrical wave in polar geometry. Based on these transformations, some analytical solutions of the wave motion in 1D inhomogeneous medium and polar inhomogeneous medium are obtained. The cases of the material properties in this paper are unreported in existing theoretical researches of inhomogeneous media dynamics.

\section{Equivalent governing equations}

The equivalent transformation relation presented in this paper is observed by constructing the equivalent relationship of the governing equations.

\subsection{Governing equation of cylindrical wave}

The undamped elastic wave motion Eq. (1) and constitutive Eq. (2) of the elastic solid are described in the tensor form as follows:

$$
\sigma_{i j, i}+\rho f_{j}=\rho \ddot{u}_{j},
$$




$$
\sigma_{i j}=c_{i j k l} u_{k, l},
$$

where $f_{j}$ is the body force, $\sigma_{i j}$ is the stress, $c_{i j k l}$ is the elastic coefficient, $u_{k, l}$ is the strain, and $\rho$ is the density.

In the absence of the body force, the equilibrium equation becomes the following form:

$$
c_{i j k l} u_{k, i l}=\rho \ddot{u}_{j} .
$$

In the cylindrical coordinate (Fig.1), the governing equation of cylindrical wave can be written as follows:

$$
\frac{1}{r} \frac{\partial}{\partial r}\left(r D \frac{\partial u}{\partial r}\right)=\rho \ddot{u},
$$

where $D=\lambda+2 \mu$ when the wave is in $\mathrm{P}$ mode; $D=\mu$ when the wave is in SH mode. $\lambda, \mu$ is Lamé coefficient. Different oscillation modes of the wave are show in Fig. 2.

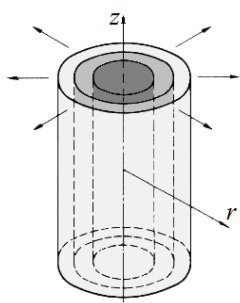

Fig. 1 Coordinate system of cylindrical wave motion

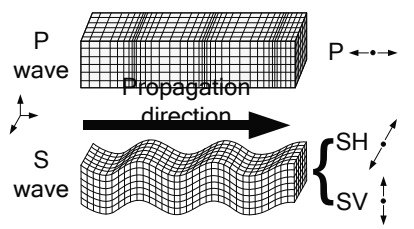

Fig. 2 Oscillation modes of the traveling wave

If the coefficient $D$ is independent on the spatial coordinate, the governing equation of the solid is described as the following equation:

$$
\frac{1}{r} \frac{\partial}{\partial r}\left(r \frac{\partial u}{\partial r}\right)=\frac{1}{c^{2}} \ddot{u},
$$

where $c$ is the elastic wave velocity of the solid medium. The elastic wave velocity of solid medium is defined by the following expression:

$$
c=\sqrt{D / \rho} .
$$

2.2. Governing equation of plane wave in bar with variable cross-section area

Consider the model of a one dimensional isotropic bar with variable cross-section area (Fig. 3). The infinitesimal body of the model is shown in Fig. 4.

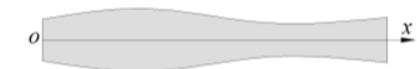

Fig. 3 Model coordinate system of the bar with crosssection area variation

Thus, the governing equation of the elastic wave in this model is given as the following form [38]:

$$
\frac{1}{A} \frac{\partial(A \sigma)}{\partial x}=\rho_{b} \ddot{u}
$$

where $A$ is the cross-section area function depended on the axial coordinate $\mathrm{x} ; \rho_{b}$ is the density of the bar.

The cylindrical wave front area of unit height is depended on radial coordinate $r$, shown as the following expression:

$$
A=2 \pi r
$$

Replace the coordinate variable $r$ in Eq. (8) with variable $x$, and substitute the modified Eq. (8) into (7), then:

$$
\frac{1}{x} \frac{\partial}{\partial x}\left(x D_{b} \frac{\partial u}{\partial x}\right)=\rho_{b} \ddot{u},
$$

where $D_{b}=E_{b}$ when the wave is in $\mathrm{P}$ mode; and $D_{b}=G_{b}$ when the wave is in S mode. $E_{b}$ is Young's modulus of the bar. $G_{b}$ is the shear elastic modulus of the bar.

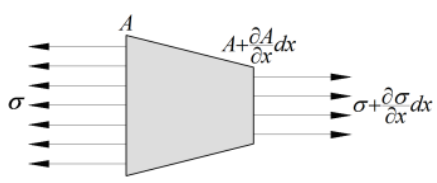

Fig. 4 Infinitesimal body of the bar with variable crosssection area

Therefore, Eq. (9) can be observed the same form of Eq. (4). When the value of $D^{\prime}$ equal to the coefficient $D$ of the solid mentioned in Section 2.1, Eqs. (9) and (4) are identical. Thus:

1. If $E_{b}=\lambda+2 \mu$ and $\rho_{b}=\rho$, the equations can be identical in $\mathrm{P}$ mode.

2. If $G_{b}=\mu$ and $\rho_{b}=\rho$, the equations can be identical in $\mathrm{S}$ mode.

Based on the above derivation, it shows that the cylindrical elastic wave equation can be equivalent to the plane wave equation of the one dimensional bar with the linear section area variation.

2.3. Governing equation of plane wave in bar with variable material properties

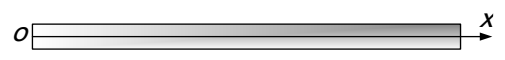

Fig. 5 Model coordinate system of the bar with material property fields

Consider another model of the one dimensional isotropic bar with the material property fields, shown in Fig. 5. The infinitesimal body of the model is shown in Fig. 6.

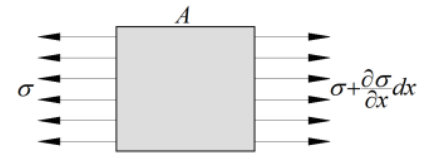

Fig. 6 Infinitesimal body of the bar with material property fields

The wave motion equation of the one dimensional bar with inhomogeneous material properties is shown as 
follows (set $A$ as the unit area):

$$
\frac{\partial}{\partial x}\left(D_{b}^{\prime} \frac{\partial u}{\partial x}\right)=\rho_{b} \ddot{u},
$$

where $D_{b}^{\prime}=E_{b}^{\prime}$ when the wave is in P mode; and $D_{b}^{\prime}=G_{b}^{\prime}$ when the wave is in S mode. $E_{b}^{\prime}$ is Young's modulus of the bar. $G_{b}^{\prime}$ is the shear elastic modulus of the bar.

Let:

$$
\begin{aligned}
& D_{b}^{\prime}=x D_{b} / x_{0}, \\
& \rho_{b}^{\prime}=x \rho_{b} / x_{0},
\end{aligned}
$$

where $x_{0}$ denotes a unit length in the direction $x$, so that $x / x_{0}$ is dimensionless. Then, Eq. (10) is transformed into Eq. (9). It reveals the equivalent transformation between the section area and material properties of the bar.

Therefore, wave motion equation of the bar with the variable section area can be equivalent to the one of the bar with material property fields, if their material property fields satisfy Eqs. (11) and (12). In consequence, the wave motion equation of the bar with material fields can also be equivalent to the cylindrical wave equation.

\section{Equivalent transformation relations}

Based on the above derivation, we can find that the equivalent cylindrical wave equation and plane wave equation have different property profile, but their wave velocity distributions are the same. Thus, one of the equivalent transformation's necessary conditions is that the problem models own the same wave velocity profile.

\subsection{Description of equivalent dynamic models}

Consider three different models satisfied these following material property fields.

Model I: Cylindrical model:

$$
\left.\begin{array}{l}
D=D(r)=\text { constant }, \\
\rho=\rho(r)=\text { constant } .
\end{array}\right\}
$$

Model II: Bar model:

$$
\left.\begin{array}{l}
D_{b}=D_{b}(x)=D(x), \\
\rho_{b}=\rho_{b}(x)=\rho(x), \\
A_{b}=A(x)=\alpha z_{0} x .
\end{array}\right\}
$$

Model III: Bar model:

$$
\left.\begin{array}{l}
D_{b}^{\prime}=\beta x D_{b} / x_{0}=\beta x D(x) / x_{0}, \\
\rho_{b}^{\prime}=\beta x \rho_{b} / x_{0}=\beta x \rho(x) / x_{0}, \\
A_{b}^{\prime}=A_{0}=\text { Unit Area },
\end{array}\right\}
$$

where $\alpha$ and $\beta$ are the undetermined coefficients; $z_{0}$ denotes unit length of direction $z$.

Thus, these three models have the same wave speed. The diagrams of their mechanical models are shown in Fig. 7.

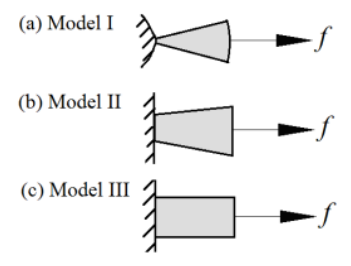

Fig. 7 Diagrams of the mechanical models

\subsection{Equivalent stiffness and mass}

In order to give the equivalent stiffness and mass, the mechanical models in Fig. 7 are all simplified to the following dynamic system (Fig. 8) which contains a spring component with equivalent stiffness coefficient $k^{e q}$ and a mass component with equivalent mass $m$.

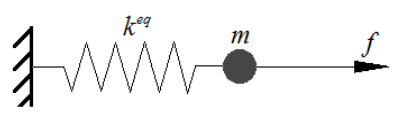

Fig. 8 Simplified dynamic system

Eqs. (16) - (18) are the formulations given for the mass of the models:

$$
\begin{gathered}
m=z_{0} \int_{S} \rho(r) d S=2 \pi z_{0} \int_{0}^{r} \rho(r) r d r=\pi r^{2} \rho z_{0}, \\
m_{b}=\int_{0}^{x} \rho_{b}(x) A(x) d x=\alpha \rho_{b} z_{0} \int_{0}^{x} x d x=\frac{1}{2} \alpha x^{2} \rho_{b} z_{0}, \\
m_{b}^{\prime}=A_{0} \int_{0}^{x} \rho_{b}^{\prime}(x) d x=\beta A_{0} \rho_{b} \int_{0}^{x} \frac{x}{x_{0}} d x=\frac{1}{2 x_{0}} \beta x^{2} \rho_{b} A_{0} .
\end{gathered}
$$

Eqs. (19) - (21) are the formulations given for generating the equivalent flexibility coefficient:

$$
\begin{aligned}
& s=\int_{0}^{r} \frac{1}{A(r) D(r)} d r=\frac{1}{2 \pi z_{0} D} \int_{0}^{r} \frac{1}{r} d r=\frac{\ln r-1}{2 \pi z_{0} D}, \\
& s_{b}=\int_{0}^{x} \frac{1}{D_{b}(x) A(x)} d x=\frac{1}{\alpha z_{0} D_{b}} \int_{0}^{x} \frac{1}{x} d x=\frac{\ln x-1}{\alpha z_{0} D_{b}}, \\
& s_{b}^{\prime}=\int_{0}^{x} \frac{1}{D_{b}^{\prime}(x) A_{0}} d x=\frac{x_{0}}{\beta D_{b} A_{0}} \int_{0}^{x} \frac{1}{x} d x=\frac{(\ln x-1) x_{0}}{\beta A_{0} D_{b}} .
\end{aligned}
$$

Thus, the equivalent stiffness coefficients can be obtained as follows:

$$
\begin{aligned}
& k^{e q}=1 / s=2 \pi z_{0} D /(\ln r-1), \\
& k_{b}^{e q}=1 / s_{b}=\alpha z_{0} D_{b} /(\ln x-1), \\
& k_{b}^{e q^{\prime}}=1 / s_{b}^{\prime}=\beta A_{0} D_{b} /(\ln x-1) x_{0} .
\end{aligned}
$$

\subsection{Determination of unknown coefficient}

The equivalent property technique presented in this paper is able to determine equivalent transformation relationships between the different mechanical models.

Let:

$$
\left.\begin{array}{l}
x_{0}=z_{0}=1, \\
x=r, A_{0}=1
\end{array}\right\}
$$


The undetermined coefficients in Eqs. (16) - (24) can be given as follows based on the rule of the same mass and the same equivalent stiffness coefficient:

$$
\begin{aligned}
& \alpha=2 \pi, \\
& \beta=2 \pi .
\end{aligned}
$$

Therefore, the transformation relation from the model I to II is shown as Eq. (28). And the relation from model I to III is shown as Eq. (29):

$$
\left.\begin{array}{rl}
D, \rho \rightarrow \begin{array}{l}
D, \rho, \\
A_{b}=2 \pi x z_{0},
\end{array} & \\
D_{b}^{\prime}=2 \pi x D / x_{0}, \\
D, \rho \rightarrow \begin{array}{c}
\rho_{b}^{\prime} \\
=
\end{array} \\
A_{b}^{\prime}=1 .
\end{array}\right\}
$$

The inverse transformations are valid as well. Where the inverse equivalent transformation from model I to III is shown as Eq. (30):

$$
\left.\left.\begin{array}{l}
D_{b}^{\prime}, \rho_{b}^{\prime} \\
A=1
\end{array}\right\} \rightarrow \begin{array}{l}
D(r)=D_{b}^{\prime} r_{0} / 2 \pi r \\
\rho(r)=\rho_{b}^{\prime} r_{0} / 2 \pi r .
\end{array}\right\}
$$

\section{Samples of analytical solutions}

The general solution forms of the equivalent wave motion equations are the same. Based on the equivalent transformation, the analytic approaches can be easily given.

\subsection{Sample I}

Consider a semi-infinite bar model (as shown in Fig. 5) with the following material property fields:

$$
\left.\begin{array}{l}
D_{b}=x \mu / x_{0}, \\
\rho_{b}=x \rho / x_{0} .
\end{array}\right\}
$$

The exact solution of the cylindrical shear wave motion in infinite homogeneous medium is written as Eq. (32), where this medium is subjected to a harmonic body force $\delta(r) e^{-i \omega t}[39]$ :

$$
W=-H_{0}^{(1)}(k r) / 4 i \mu,
$$

where the wave number $k=\omega / c=\sqrt{\rho \omega^{2} / \mu}$.

Based on the equivalent transformation relation, the corresponding exact solution of the bar model can be given directly:

$$
W=-\pi H_{0}^{(1)}(k x) / 2 i \mu \text {. }
$$

\subsection{Sample II}

Focus on an infinite inhomogeneous medium with the polar geometry and variable material properties (Fig. 9). The material property distributions are defined as the following form:

$$
\left.\begin{array}{l}
D=\xi r_{0} / r \\
\rho=\zeta r_{0} / r
\end{array}\right\}
$$

where $r_{0}$ is the unit length in direction $r ; \xi$ and $\zeta$ are constant.

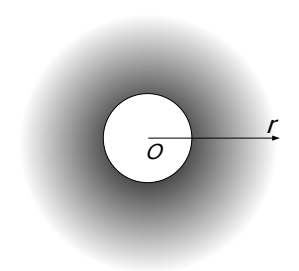

Fig. 9 Model of sample II

Based on the inverse equivalent transformation, the distribution of equivalent shear modulus and density are determined for the bar model with constant section area, shown as Eq. (35):

$$
\left.\begin{array}{l}
D_{b}=2 \pi \xi, \\
\rho_{b}=2 \pi \zeta .
\end{array}\right\}
$$

The equivalent material properties (Eq. (35)) shows that the equivalent bar model with constant section area is homogeneous. Assume a harmonic wave $e^{-i \omega t}$ propagating form $r_{1}$ to $+\infty$ along the axis $r$. Thus, the analytic approach of displacement field (Eq. (36)) is given:

$$
W=e^{-i\left[\omega t-k\left(r-r_{1}\right)\right]},
$$

where $k=\omega / c=\sqrt{\zeta \omega^{2} / \xi}$ is the wave number.

\subsection{Sample III}

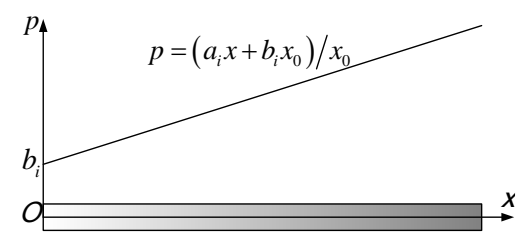

Fig. 10 Model of sample III

Pay attention to a semi-infinite inhomogeneous bar with the constant section area and material property fields as follows, shown in Fig. 10:

$$
\left.\begin{array}{l}
D_{b}=\left(a_{1} x+b_{1} x_{0}\right) / x_{0}, \\
\rho_{b}=\left(a_{2} x+b_{2} x_{0}\right) / x_{0},
\end{array}\right\}
$$

where $a_{1}$ and $b_{1}$ are constant parameters of shear modulus term; $a_{2}$ and $b_{2}$ are constant parameters of density term.

A harmonic wave $e^{-i \omega t}$ is propagating from 0 to $\infty$ along the axis $x$. In the process of wave propagation, no reflection or diffraction will happen. Then, some analytical approaches of this problem can be given:

1. When $a_{i}=0$ and $b_{i}>0$, the analytical dis- 
placement field is shown as Eq. (39). In this situation, the bar model is homogeneous:

$$
W=e^{-i(\omega t-k x)}=e^{-i \omega\left(t-\sqrt{b_{2} / b_{1}} x\right)} .
$$

2 When $a_{i}>0, b_{i} \geq 0$, define another coordinate system $x^{\prime}$ obtained by coordinate transformation as follows. Fig. 11 shows this situation:

$$
x^{\prime}=x+b_{1} x_{0} / a_{1}
$$

Then,

$$
\left.\begin{array}{l}
D_{b}\left(x^{\prime}\right)=a_{1} x^{\prime} / x_{0}, \\
\rho_{b}\left(x^{\prime}\right)=a_{2} x^{\prime} / x_{0} .
\end{array}\right\}
$$

Thus, in the equivalent cylindrical wave motion model, equivalent shear modulus is $a_{1} / 2 \pi$; equivalent density is $a_{2} / 2 \pi$.

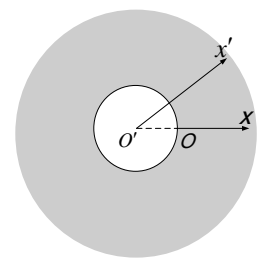

Fig. 11 Equivalent model of sample III when $a_{i}>0$ and $b_{i} \geq 0$

Therefore, the analytic solution is as Eq. (42):

$$
W=H_{0}^{(1)}\left(k x^{\prime}\right) e^{-i \omega t} / H_{0}^{(1)}\left(k b_{1} x_{0} / a_{1}\right)
$$

3. When $a_{i}<0$ and $b_{i}>0$, define another coordinate system $x^{\prime}$ obtain by coordinate transformation as follows. Fig. 12 shows this situation:

$$
x^{\prime}=-x-b_{1} x_{0} / a_{1} .
$$

Then,

$$
\left.\begin{array}{l}
D_{b}\left(x^{\prime}\right)=-a_{1} x^{\prime} / x_{0}, \\
\rho_{b}\left(x^{\prime}\right)=-a_{2} x^{\prime} / x_{0} .
\end{array}\right\}
$$

Thus, in the equivalent cylindrical wave motion model, equivalent shear modulus is $-a_{1} / 2 \pi$; equivalent density is $-a_{2} / 2 \pi$.

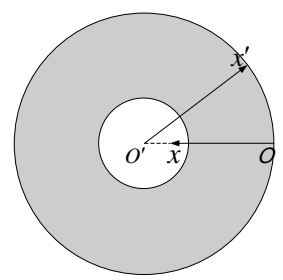

Fig. 12 Equivalent model of model III when $a_{i}<0$ and $b_{i} \geq 0$

Therefore, the analytical displacement solution is shown as Eq. (45). Because of singularity at $x=-b_{1} x_{0} / a_{1}$, the valid domain of this solution is $x \in\left[0,-b_{1} x_{0} / a_{1}\right)$ :

$$
W=H_{0}^{(1)}\left(k x^{\prime}\right) e^{-i \omega t} / H_{0}^{(1)}\left(-k b_{1} x_{0} / a_{1}\right) .
$$

\subsection{Sample IV}

An infinite 1D inhomogeneous medium with constant section area is taken into account (Fig. 13).

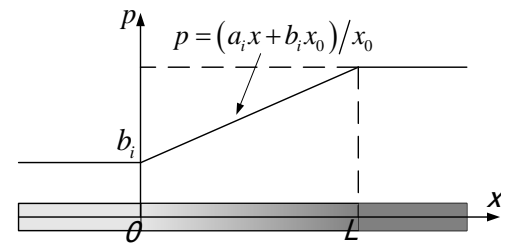

Fig. 13 Model of sample IV

This model consists of three parts. The left part and right part are homogeneous. The middle part is inhomogeneous, where the length is $L$. Then, the material property fields are continuous in domain defined in this sample, where the functions of material property are denoted as the following expressions:

$$
\begin{aligned}
& D_{b}= \begin{cases}b_{1}, & -\infty<x<0, \\
\left(a_{1} x+b_{1} x_{0}\right) / x_{0}, & 0 \leq x \leq L, \\
\left(a_{1} L+b_{1} x_{0}\right) / x_{0}, & L<x<+\infty,\end{cases} \\
& \rho_{b}= \begin{cases}b_{2}, & -\infty<x<0, \\
\left(a_{2} x+b_{2} x_{0}\right) / x_{0}, & 0 \leq x \leq L, \\
\left(a_{2} L+b_{2} x_{0}\right) / x_{0}, & L<x<+\infty,\end{cases} \\
& \left.\begin{array}{l}
a_{1} b_{2}=a_{2} b_{1}, \\
\left(a_{1} L+b_{1} x_{0}\right) / x_{0}>0 \\
\left(a_{2} L+b_{2} x_{0}\right) / x_{0}>0
\end{array}\right\}
\end{aligned}
$$

where $a_{1}$ and $b_{1}$ are constant parameters of shear modulus term; $a_{2}$ and $b_{2}$ are constant parameters of density term. Thus, the wave numbers of the left, middle and right part are all equal.

A harmonic wave is propagating from $-\infty$ to $+\infty$ along the axis $x$. The dynamic response is observed at $x=0$ as the following form, where: $\varphi_{0}$ denotes initial phase:

$$
\left.W\right|_{x=0}=e^{-i\left(\omega t+\varphi_{0}\right)}
$$

Because the material parameter functions of this sample are continuous in the definition domain, there is no reflection or diffraction when the wave is propagating. Then, the analytical approaches of this problem can be given based on the above mentioned derivation.

1. On the left part $(x<0)$, traveling wave keeps constant amplitude. Thus:

$$
\left.W\right|_{x<0}=e^{-i\left(\omega t+\varphi_{0}+k x\right)}
$$

2. On the middle part $(0<x \leq L)$, the value of $a_{1}$ 
and $a_{2}$ need be discussed. Then, the dynamic response at $x=L$ can be given. And the response on the right part $(0<x \leq L)$ is able to determined:

a) When $a_{i}=0$, the middle part and right part are the same homogeneous medium. Thus:

$$
\left.W\right|_{x \geq 0}=e^{-i\left(\omega t+\varphi_{0}-k x\right)} .
$$

b) When $a_{i}>0$, the dynamic response on the middle part is shown as follows:

$$
\begin{aligned}
& \left.W\right|_{0 \leq x \leq L}=\frac{H_{0}^{(1)}\left(k x+b_{1} k x_{0} / a_{1}\right)}{H_{0}^{(1)}\left(b_{1} k x_{0} / a_{1}\right)} e^{-i\left(\omega t+\varphi_{0}\right)}, \\
& \left.W\right|_{x>L}=\frac{H_{0}^{(1)}\left(k L+b_{1} k x_{0} / a_{1}\right)}{H_{0}^{(1)}\left(b_{1} k x_{0} / a_{1}\right)} e^{-i\left[\omega t+\varphi_{0}-k(x-L)\right]} .
\end{aligned}
$$

c) When $a_{i}<0$, the dynamic response on the middle part is shown as follows:

$$
\begin{aligned}
& \left.W\right|_{0 \leq x \leq L}=\frac{H_{0}^{(1)}\left(-k x-b_{1} k x_{0} / a_{1}\right)}{H_{0}^{(1)}\left(-b_{1} k x_{0} / a_{1}\right)} e^{-i\left(\omega t+\varphi_{0}\right)}, \\
& \left.W\right|_{x>L}=\frac{H_{0}^{(1)}\left(-k L-b_{1} k x_{0} / a_{1}\right)}{H_{0}^{(1)}\left(-b_{1} k x_{0} / a_{1}\right)} e^{-i\left[\omega t+\varphi_{0}-k(x-L)\right]} .
\end{aligned}
$$

\subsection{Sample V}

An infinite polar geometry (Fig. 9) with the polar inhomogeneity (Eq. (56)) is considered in this sample:

$$
\left.\begin{array}{l}
D=r_{0} /\left(a_{1} r+b_{1} r_{0}\right), \\
\rho=r_{0} /\left(a_{2} r+b_{2} r_{0}\right),
\end{array}\right\}
$$

where $a_{1}, a_{2}, b_{1}$ and $b_{2}$ are all constant, and $a_{1} b_{2}=a_{2} b_{1}$.

A harmonic wave $e^{-i \omega t}$ is propagating from $r_{1}$ to $\infty$ along the axis $r$. Some approaches can be given:

1. When $a_{i}=0$ and $b_{i}>0$, the medium is homogeneous, where: $D=1 / b_{1}$, and $\rho=1 / b_{2}$. Thus, the displacement field can be obtained based on Eq. (32):

$$
W=H_{0}^{(1)}(k r) e^{-i \omega t} / H_{0}^{(1)}\left(k r_{1}\right) .
$$

2. When $a_{i}>0, b_{i} \geq 0$, define another coordinate system $r^{\prime}$ by the coordinate transformation Eq. (58):

$$
r^{\prime}=r+b_{1} r_{0} / a_{1}
$$

Then, the equivalent elastic modulus and density of the bar model with constant section area are determined:

$$
\left.\begin{array}{rl}
G_{b} & =2 \pi / a_{1} \\
\rho_{b} & =2 \pi / a_{2}
\end{array}\right\}
$$

Thus, the analytic solution of displacement field can be given based on Eq. (60):

$$
W=e^{-i\left[\omega t-k\left(r-r_{1}\right)\right]} .
$$

3. When $a_{i}<0, b_{i}>0$, define another coordinate system $r^{\prime}$ by the coordinate transformation Eq.(61):

$$
r^{\prime}=-r-b_{1} r_{0} / a_{1}
$$

Then, equivalent modulus and density of the bar model with constant section area are determined:

$$
\left.\begin{array}{l}
G_{b}=-2 \pi / a_{1}, \\
\rho_{b}=-2 \pi / a_{2} .
\end{array}\right\}
$$

Therefore, the analytical solution of displacement field is the same as Eq. (60). However, the material property has singularity at $r=-b_{1} r_{0} / a_{1}$.

\section{Conclusions}

The equivalent transformation relations are presented in this paper, which are between plane wave in onedimensional media and the cylindrical wave in polar geometry. By the theoretical analysis for the equivalent relations, it is proved that the cylindrical wave problem can be transformed into the plane wave in bar with variable section area or material properties. Inverse transformations are valid too. Applying these transformations, the unknown solutions are given directly based on the known solutions of the equivalent models. Five different samples of the wave motion problems in the inhomogeneous media are solved and discussed, where the studied material property distributions are unreported in the published theoretical researches of inhomogeneous media dynamics.

The nature of presented equivalent transformation relations is transformation between geometric shape and material properties. In prospect, the presented equivalent property technique will help to develop the theories of acoustic, electromagnetic, elastic and seismic waves.

\section{Acknowledgements}

The work was supported by the Natural Science Foundation of Heilongjiang Province (A201310) and the Scientific Research Starting Foundation for Post Doctorate of Heilongjiang Province (LBH-Q13040).

\section{References}

1. Mow, C.C.; Pao, Y.H. 1973. Diffraction of elastic waves and dynamic stress concentrations. Washington: Crane, Russak.

2. Kim, J.H.; Paulino, G.H. 2002. Isoparametric graded finite elements for nonhomogeneous isotropic and orthotropic materials, Journal of Applied Mechanics 69: 502-514 http://dx.doi.org/10.1115/1.1467094.

3. Wang, C.D.; Lin, Y.T.; Jeng, Y.S.; Ruan, Z.W. 2010. Wave propagation in an inhomogeneous crossanisotropic medium, International Journal for Numerical and Analytical Methods in Geomechanics 34: 711732.

http://dx.doi.org/10.1002/nag.826. 
4. Pao, Y.H.; Su, X.Y.; Tian, J.Y. 2000. Reverberation matrix method for propagation of sound in a multilayered liquid, Journal of Sound and Vibration 230: 743-760. http://dx.doi.org/10.1006/jsvi.1999.2675.

5. Virieux, J. 1984. SH-wave propagation in heterogeneous media: velocity-stress finite-difference method, Geophysics 49(11): 1933-1957. http://dx.doi.org/10.1190/1.1441605.

6. Virieux, J. 1986. P-SV wave propagation in heterogeneous media: Velocity-stress finite-difference method, Geophysics 51(4): 889-901. http://dx.doi.org/10.1190/1.1442147.

7. Oyekoya, O.O.; Mba, D.U.; El-Zafrany, A.M. 2009. Buckling and vibration analysis of functionally graded composite structures using the finite element method, Composite Structures 89(1): 134-142. http://dx.doi.org/10.1016/j.compstruct.2008.07.022.

8. Yang, Z.L.; Wang, Y.; Hei, B.P. 2013. Transient analysis of 1D inhomogeneous media by dynamic inhomogeneous finite element method, Earthquake Engineering and Engineering Vibration 12(4): 569-576. http://dx.doi.org/10.1007/s11803-013-0198-5.

9. Wang, Y.; Yang, Z.L.; Hei, B.P. 2013. An investigation on the displacement response in one-dimension inhomogeneous media under different loading speeds. Journal of Northeastern University, 34(S2): 18-21 (in Chinese).

10. Zienkiewicz, O.C.; Taylor, R.L. 2000. The finite element method (fifth edition) volume 3: Fluid dynamics. London: Butterworth-Heinemann.

11. Kucukcoban, S.; Kallivokas, L.F. 2011. Mixed perfectly-matched-layers for direct transient analysis in 2D elastic heterogeneous media, Comput. Methods Appl. Mech. Engrg. 200: 57-76.

http://dx.doi.org/10.1016/j.cma.2010.07.013.

12. Wilson, J.T. 1942. Surface waves in a heterogeneous medium, Bulletin of the Seismological Society of America 32: 297-304.

13. Vrettos, C. 1990. In-plane vibrations of soil deposits with variable shear modulus: I. surface waves, International journal for Numerical and Analytical Methods in Geomechanics 14(3): 209-222. http://dx.doi.org/10.1002/nag.1610140304.

14. Vrettos, C. 1990. In-plane vibrations of soil deposits with variable shear modulus: II. Line load, International Journal for Numerical and Analytical Methods in Geomechanics 14(9): 649-662. http://dx.doi.org/10.1002/nag.1610140905.

15. Vrettos, C. 1990. Dispersive SH-surface waves in soil deposits of variable shear modulus, Soil Dynamics and Earthquake Engineering 9(5): 255-264. http://dx.doi.org/10.1016/S0267-7261(05)80004-1.

16. Leung, K.L.; Vardoulakis, I.G.; Beskos, D.E.; Tassoulas, J.L. 1991. Vibration isolation by trenches in continuously nonhomogeneous soil by the BEM, Soil Dynamics and Earthquake Engineering 10(3): 172179. http://dx.doi.org/10.1016/0267-7261(91)90030-4.

17. Stoneley, R. 1934. The transmission of Rayleigh waves in a heterogeneous medium, Geophysical Journal International 3(S6): 222-232.

http://dx.doi.org/10.1111/j.1365-246X.1934.tb01735.x.

18. Newlands, M. 1950. Rayleigh waves in a two-layer heterogeneous medium, Geophysical Journal International 6(S2): 109-124.

http://dx.doi.org/10.1111/j.1365-246X.1950.tb02985.x.

19. Vardoulakis, I. 1984. Torsional surface waves in inhomogeneous elastic media, International Journal for Numerical and Analytical Methods in Geomechanics 8(3): 287-296.

http://dx.doi.org/10.1002/nag.1610080306.

20. Ben-Menahem, A. 1960. Diffraction of elastic waves from a surface source in a heterogeneous medium. Bulletin of the Seismological Society of America 50(1): 15-33.

21. Hudson, J.A. 1962. Love waves in a heterogeneous medium, Geophysical Journal of the Royal Astronomical Society 6(2): 131-147.

http://dx.doi.org/10.1111/j.1365-246X.1962.tb02976.x.

22. Hook, J.F. 1961. Separation of the vector wave equation of elasticity for certain types of inhomogeneous, isotropic media, Journal of the Acoustical Society of America 33(3): 302-313. http://dx.doi.org/10.1121/1.1908646.

23. Deresiewicz, H. 1962. A note on Love waves in a homogeneous crust overlying an inhomogeneous substratum, Bulletin of the Seismological Society of America 52(3): 639- 645.

24. Gazetas, G. 1980. Static and dynamic displacements of foundations on heterogeneous multilayered soils, Géotechnique 30(2): 159-177.

http://dx.doi.org/10.1680/geot.1980.30.2.159.

25. Watanabe, K. 1982. Transient response of an inhomogeneous elastic solid to an impulsive SH-source (variable SH-wave velocity), Bulletin of the JSME 25(201): 315-320. http://dx.doi.org/10.1299/jsme1958.25.315.

26. Manolis, G.D.; Bagtzoglou, A.C. 1992. A numerical comparative study of wave propagation in inhomogeneous and random media. Computational Mechanics 10(6): 397-413 http://dx.doi.org/10.1007/BF00363995.

27. Watanabe, K.; Payton, R.G. 2004. Green's function and its non-wave nature for $\mathrm{SH}$-wave in inhomogeneous elastic solid, International Journal of Engineering Science 42(19-20): 2087-2106.

http://dx.doi.org/10.1016/j.ijengsci.2004.08.001.

28. Pekeris, C.L. 1935. The propagation of Rayleigh waves in heterogeneous media, J. Appl. Phys. 6: 133138. http://dx.doi.org/10.1063/1.1745304.

29. Shaw, R.P.; Makris, N. 1992. Green's functions for Helmholtz and Laplace equations in heterogeneous media, Engineering Analysis with Boundary Elements 10(2): 179-183. http://dx.doi.org/10.1016/0955-7997(92)90049-D.

30. Dey, S.; Gupta, A.K.; Gupta, S. 1996. Propagation of Rayleigh waves in a heterogeneous incompressible substratum over a homogeneous incompressible halfspace. International Journal for Numerical and Analytical Methods in Geomechanics 20(5): 365-375.

http://dx.doi.org/10.1002/(SICI)1096-

9853(199605)20:5<365::AID-NAG823>3.0.CO;2-0.

31. Boutin, C.; Auriault, J.L. 1993. Rayleigh Scattering in Elastic Composite Materials, Int. J. Eng. Sci. 31(12): 1669-1689.

http://dx.doi.org/10.1016/0020-7225(93)90082-6. 
32. Chen, W.; Fish, J. 2001. A dispersive model for wave propagation in periodic heterogeneous media based on homogenization with multiple spatial and temporal scales, Journal of Applied Mechanics 68(2): 153-161. http://dx.doi.org/10.1115/1.1357165.

33. Charalambakis, N. 2010. Homogenization Techniques and Micromechanics: A Survey and Perspectives, Applied Mechanics Reviews 63(3): 030803. http://dx.doi.org/10.1115/1.4001911.

34. Fish, J.; Filonova, V.; Kuznetsov, S. 2012. Microinertia effects in nonlinear heterogeneous media, International Journal for Numerical Methods in Engineering, 91(13): 1406-1426. http://dx.doi.org/10.1002/nme.4322.

35. Pendry, J.B.; Schurig, D.; Smith, D.R. 2006. Controlling electromagnetic fields, Science 312(5781): 1780-1782. http://dx.doi.org/10.1126/science.1125907.

36. Zhou, X.M.; Hu, G.K.; Lu, T.J. 2008. Elastic wave transparency of a solid sphere coated with metamaterials, Physical Review B 77(2): 024101. http://dx.doi.org/10.1103/PhysRevB.77.024101.

37. McManus, T.M.; Valiente-Kroon, J.A.; Horsley, S.A.R.; Hao, Y. 2014. Illusions and cloaks for surface waves, Scientific Reports 4: 5977. http://dx.doi.org/10.1038/srep05977.

38. Wang, L.L. 2006. Foundations of Stress Waves. Elsevier Science Ltd.

39. Pao, Y.H. 1983. Elastic waves in solids, Journal of applied mechanics 50(4b): 1152-1164. http://dx.doi.org/doi:10.1115/1.3167197.
Y. Wang, Z.L. Yang, J.W. Zhang

THEORY AND APPLICATION OF THE EQUIVALENT TRANSFORMATION RELATIONSHIP BETWEEN PLANE WAVE IN ONE-DIMENSIONAL MEDIUM AND CYLINDRICAL WAVE IN POLAR GEOMETRY

\section{S u m m a r y}

Based on the equivalent equation and property technique, the equivalent transformation relationship is established, which is between the plane wave in the one dimensional inhomogeneous medium and the cylindrical wave in the polar geometry. The nature of presented equivalent transformation relations is the transformation between polar geometry and material properties. It is proved that the cylindrical wave problem is able to be transformed into plane wave in bar with variable section area or variable material properties. Five different samples of wave motion problems in the inhomogeneous media are solved and discussed, where the studied material property distributions are unreported in the published theoretical researches of inhomogeneous media dynamics. The exact approaches of these samples are presented in present paper.

Keywords: equivalent transformation, analytical solution, inhomogeneous medium, wave motion, polar geometry.

Received December 18, 2014 Accepted January 15, 2015 